on their ultimate outcome. Previous work has found that a high percentage of older psychiatric in-patients $(50 \%-60 \%)$ had a personality disorder. Others have also confirmed a poorer outcome of axis I disorders in personality-disordered clients. However, this could vary widely resulting from different sample characteristics, different measures and different diagnostic criteria. The presence of personality disorders has also been shown to adversely affect relationships, which may also be deleterious to recovery, as supportive relationships improve/hasten recovery. Our hypothesis is that those elderly psychiatric in-patients with comorbid personality disorders will take longer to treat, and their recovery will be less complete than those without personality disorders. A single case series study of adult psychiatric in-patients is being undertaken to determine the prevalence of personality disorder and the effect on treatment of their axis I disorder. This study will contribute to our knowledge of the effects of personality disorder in elderly psychiatric in-patients and should provide clues as to how these conditions could be managed to affect improved outcomes.

02-07

\section{The effect of personality disorder on treatment for depression: psychotherapy vs. medication}

\section{RT Mulder, PR Joyce, SE Luty, CM Frampton, JD Carter}

Department of Psychological Medicine, Christchurch School of Medicine \& Health Sciences, University of Otago, Christchurch, New Zealand

Aims: To study the impact of comorbid personality disorders (PDs) on outcome in 1) a medication trial for major depression (MD) and 2) a psychotherapy trial for MD.

Method: Two treatment trials for patients with MD were performed: 1) randomized clinical trial of fluoxetine vs. nortriptyline with open follow-up $(n=195)$ and 2) randomized clinical trial of interpersonal psychotherapy (IPT) vs. cognitive behavioural therapy $(n=177)$.

Results: Comorbid PD had no impact on the outcome of MD in patients treated with medication over the short term. Avoidant PD was associated with poorer outcome over the longer term. Comorbid PD had a negative impact on the outcome of MD in patients treated with psychotherapy over the short term. This was largely explained by the poor response to IPT in depressed patients with comorbid PD.
Discussion: The finding that comorbid PD adversely affects the outcome in depressed patients treated with psychotherapy but not with drugs is consistent with other studies. Possible mechanisms are discussed.

02-08

\section{A randomized controlled trial of psychotherapeutic early intervention for borderline personality disorder}

\author{
A Chanen ${ }^{1,2}$, H Jackson ${ }^{1,3}$, L McCutcheon ${ }^{1,2}$, \\ D Germano ${ }^{2}$, H Nistico², P Dudgeon ${ }^{3}$, HP Yuen', \\ E McDougall', C Weinstein', V Clarkson', P McGorry ${ }^{1,2}$
}

'ORYGEN Research Centre, Department of Psychiatry, The University of Melbourne; ${ }^{2}$ ORYGEN Youth Health, Melbourne Health; and ${ }^{3}$ School of Behavioural Science,

The University of Melbourne, Melbourne, Australia

Background: Borderline personality disorder (BPD) is a major public health problem. This study aims to delay the onset of or reduce the severity of subsequent BPD in a sample of adolescents at risk of BPD.

Method: Eighty-six 15- to 18-year-old participants were drawn from an adolescent psychiatric service. They had one or more childhood risk factors for BPD and met criteria for two or more current DSM-IV BPD features, using rigorous diagnosis. Participants were randomly allocated to 24 sessions of either cognitive analytic therapy [CAT; Ryle 1997] or manualized 'good clinical care' (GCC). A third, nonrandomized comparison group $(n=30)$, which received 'treatment as usual' (TAU), was collected prior to implementation of the study. Outcome measures completed at baseline, 6, 12 and 24 months included BPD score, internalizing and externalizing psychopathology and social and occupational functioning.

Results: At 24 months, patients receiving CAT had statistically significant lower levels of externalizing pathology compared with those receiving GCC. Patients receiving CAT had statistically significant lower scores on internalizing and externalizing psychopathology compared with those receiving TAU, and patients receiving GCC had statistically significant lower scores on internalizing psychopathology compared with TAU. BPD scores improved from baseline levels in all three conditions and no significant differences were found between the groups.

Conclusion: CAT is an effective early intervention for BPD and is superior to both manual-based ' $\mathrm{GCC}$ ' and TAU. 